[0212-7199 (2004) 21: 2; pp 62-68] ANALES DE MEDICINA INTERNA Copyright () 2004 ARAN EDICIONES, S.L.

An. MED. InTERnA (Madrid) Vol. 21, N. ${ }^{\circ} 2$, pp. 62-68, 2004

\title{
Vigilancia epidemiológica de la intoxicación aguda en el Área Sur de la Comunidad de Madrid: Estudio VEIA 2000
}

\author{
P. J. CABALLERO VALLÉS ${ }^{1}$, S. DORADO POMBO, B. JEREZ BASURCO, \\ M. MEDINA SAMPEDRO, B. BRUSÍNT OLIVARES
}

Hospital Universitario 12 de Octubre. ${ }^{\text {} C e n t r o ~ d e ~ S a l u d ~ " A ́ n g e l a ~ U r i a r t e " . ~ M a d r i d ~}$

EPIDEMIOLOGIC SURVEY OF ACUTE POISONING IN THE SOUTH AREA OF THE COMMUNITY OF MADRID. THE VEIA 2000 STUDY

\section{RESUMEN}

Objetivo: Extensos estudios observacionales sobre intoxicaciones agudas (IA) permiten detectar cambios de tendencia imprescindibles para diseñar actuaciones preventivas. VEIA es un estudio evolutivo de las IA atendidas en Urgencias del Hospital 12 de Octubre de Madrid en periodos anuales completos $[1979(1,2), 1985$ (3), 1990 (4), 1994 (5) y 1997 (6)], presentamos el 2000 y comparamos los resultados con los previos.

Métodos y resultados: El método se mantiene idéntico. 1.128 IA $88 \%$ voluntarias. 451 intentos de suicidio. Las benzodiazepinas son el $44 \%$ de los medicamentos; El alcohol el 75\% de los tóxicos no farmacológicos y las drogas el $19 \%$.

Conclusiones: En las mujeres aumentan las IA por alcohol y drogas y disminuyen los intentos de suicidio, ello supone una aproximación de los roles hombre/mujer. Hay un envejecimiento en los IS (¿se "pasa de moda" suicidarse entre los jóvenes?) y advertimos dos pautas: IS con medicamentos con $0,1 \%$ de mortalidad y por no-medicamentos con $3 \%$. Dos de cada cinco hombres con IS son adictos.

Disminuyen las benzodiazepinas, el paracetamol sustituye a la Aspirina y los AINE a los restantes analgésicos. El alcohol es el tóxico no-farmacológico predominante, pero disminuye un $11 \%$. Las drogas, que ahora superan a los venenos tradicionales (gases, disolventes, etc.) suponen un 40\% más que en 1997. Al comparar 1994 y 2000, la heroína no cambia pero la cocaína pasa de 13 casos a 67 y las anfetaminas tipo MDMA crecen de modo geométrico.

PALABRAS CLAVE: Intoxicación aguda. Epidemiología. Intentos de suicidio. Intoxicación medicamentosa. Intoxicación alcohólica. Intoxicación por drogas.

\begin{abstract}
Objective: Extensive observational studies of acute intoxications (AI) allow the detection of trend changes indispensable for the design of preventive actions. VEIA is an evolutional study of AI attended at the Emergency Services of the Hospital "Doce de Octubre" of Madrid over all-round annual periods (1979, 1985, 1990, 1994 and 1997); we present the results of 2000 and compare them with those of previous years.

Methods and results: An identical method was used. The hospital attended 1,128 AI, 88\% of them voluntary. There were 451 suicide attempts. Forty four per cent of drugs involved were benzodiazepines. Alcohol represented $75 \%$ of non-pharmacological toxic substances and drugs, $19 \%$.

Conclusions: An increase of AI caused by alcohol and drugs was observed among women, as well as a decrease of suicide attempts, which reflects an approximation of man/woman roles. An aging trend was observed in suicide attempts (Is suicide "outmoded" among young people?), along with two patterns: Suicide attempts with drugs had a mortality rate of $0.1 \%$ and suicide attempts without drugs, of $3 \%$. Two out of five men attempting suicide had drug addictions.

There had been a reduction of benzodiazepines use and substitution of aspirine for paracetamol and of other analgesics for NSAID. Alcohol was the predominant non-pharmacological toxic substance, but had decreased $11 \%$. Drugs, that had surpassed the traditional poisons (gases, solvents, etc.) represented 40\% more than in 1997. When 1994 and 2000 were compared, heroine had not changed significantly, but cocaine had increased from 13 cases to 67 and amphetamines type MDMA had increased geometrically.
\end{abstract}

KEY WORDS: Acute intoxication. Epidemiology. Suicide attempts. Medicine intoxication. Alcohol intoxication. Drug intoxication.

Caballero Vallés PJ, Dorado Pombo S, Jerez Basurco B, Medina Sampedro M, Brusínt Olivares B. Vigilancia epidemiológica de la intoxicación aguda en el Área Sur de la Comunidad de Madrid: Estudio VEIA 2000. An Med Interna (Madrid) 2004; 21: 62-68.

\section{INTRODUCCIÓN}

Las IA tienen unas características epidemiológicas básicamente uniformes, a pesar de lo cual se observan patrones variables y tendencias dinámicas que, a modo de "oleadas", evolucionan a lo largo del tiempo (1-11) y coinciden cuando se comparan áreas socio-económicas similares; trabajos publicados en España (12-23) concuerdan en buena medida con nuestros hallazgos de estos años (1-6).

El estudio cronológico de las IA refleja tanto los cambios sanitarios -las pautas de prescripción, el abuso de tranquilizantes, los modernos antidepresivos, las drogas emergentes, 
etc.- como las actuaciones políticas -el Prosereme (24)- o la variabilidad social que en la actualidad suaviza las tradicionales diferencias por género o hábitat (25).

Otro aspecto interesante de estos estudios evolutivos se demuestra, además, porque la detección de cambios en las tendencias de un país, puede servir de indicador para otros y las disposiciones adoptadas suelen ser igualmente eficaces en unos y otros. Tampoco debemos olvidar que el elevado costo económico (26) y de vidas puede atenuarse con medidas preventivas deducidas de este tipo de estudios (27). El aumento progresivo de intentos de suicidio (IS) en hombres (28), la elevada proporción de muertes extrahospitalarias (26-29) o la creciente reincidencia en los IS (21), indican lo mucho que queda por realizar y ha conducido a que la OMS en su programa "Salud para todos en el 2000", haya propuesto intentar invertir la tendencia creciente de los IS (30).

En nuestra última publicación (6) propusimos el concepto de "Vigilancia epidemiológica de las intoxicaciones agudas" para explicitar esta versatilidad y en la actual nos proponemos darle mayor entidad y garantizar su continuidad, además de facilitar la presentación de los resultados, hasta ahora obligatoriamente globales y constreñidos a una única publicación. Nuestro compromiso es mantener estos estudios periódicos y ampliar aspectos puntuales que de ellos se derivan.

\section{MATERIAL Y MÉTODOS}

Se han recogido y estudiado los casos de IA atendidos en la Sección de Urgencias del Hospital 12 de Octubre de Madrid durante el año 2000. Este centro presta asistencia sanitaria al Área 11 del INSALUD de la Comunidad de Madrid y es a la vez Hospital de referencia de otros centros sanitarios, por lo que proporciona asistencia a 665.196 personas mayores de catorce años. El número de urgencias atendidas (excluidas las pediátricas y ginecológicas) fue de 166.577 (63.592 médicas) y 1.128 correspondieron a IA.

La metodología es igual a la de anteriores publicaciones (2-6) por lo que denominamos intoxicación al contacto de productos tóxicos potenciales: a) medicamentos: cuando superan la dosis máxima terapéutica, incluso si intentan mitigar un síntoma y se ingiere una cantidad excesiva (sobredosificaciones); b) alcohol y drogas: cuando interfieren una actividad mínimamente normal, por ejemplo el equilibrio; y c) otros tóxicos, a cualquier dosis.

Dividimos las IA en voluntarias (IAV) (intentos de suicidio, alcohólicas, drogas y sobredosificaciones, según la intención) y accidentales (IAA) (laborales o domésticas, dependiendo del lugar donde ocurren). Al igual que en trabajos anteriores (2-6) se excluyen las toxiinfecciones alimentarias y las yatrogénicas.

Los tóxicos se clasifican en: medicamentos y no medicamentos. Hablamos de fármacos o medicamentos para referirnos a los principios activos y de presentaciones farmacéuticas para denominar los distintos tipos de preparados comerciales empleados por cada paciente.

Hemos analizado la distribución de los casos según la edad, sexo, tipo de intoxicación y tóxico. También se han estudiado los antecedentes, así como su posterior derivación y la adscripción o no del paciente al área de cobertura del hospital. Se trata de un estudio descriptivo transversal en el que se han empleado técnicas básicas de estadística descriptiva.
Cada grupo se define por la proporción sobre el total en variables cualitativas y la media, mediana y/o moda, así como desviación estándar para las cuantitativas. Para la comparación de las medias se utilizó la prueba de Student, el límite de significación se consideró $\mathrm{p}<0,05$ y el intervalo de confianza del $95 \%$.

\section{RESULTADOS}

\section{CARACTERÍSTICAS GENERALES}

Se atendieron 1.128 IA (170/100.000 habitantes), correspondientes al $1,77 \%$ de las urgencias médicas y el $0,68 \%$ de las totales. El número de mujeres es $540(48 \%)$ y 588 los hombres (52\%), con una proporción $\mathrm{M} / \mathrm{H}$ de 1:1,08.

Las edades oscilaron entre 15 y 90 años, con una media \pm desviación estándar para el conjunto de la serie de $37 \pm 15$ años (mediana y moda 33 ), $37 \pm 15$ para los hombres (mediana 35) y $35 \pm 15,5$ para las mujeres (mediana 32 ); la diferencia es significativa al comparar la edad media entre los sexos (error estándar: 0,91, p<0,05). La distribución por edades y género, así como la pirámide poblacional del Área 11 del INSALUD de la Comunidad de Madrid, según el padrón de 1996 se expone en la figura 1. Un $15 \%$ de los pacientes atendidos no pertenecían a la zona de influencia del Hospital.

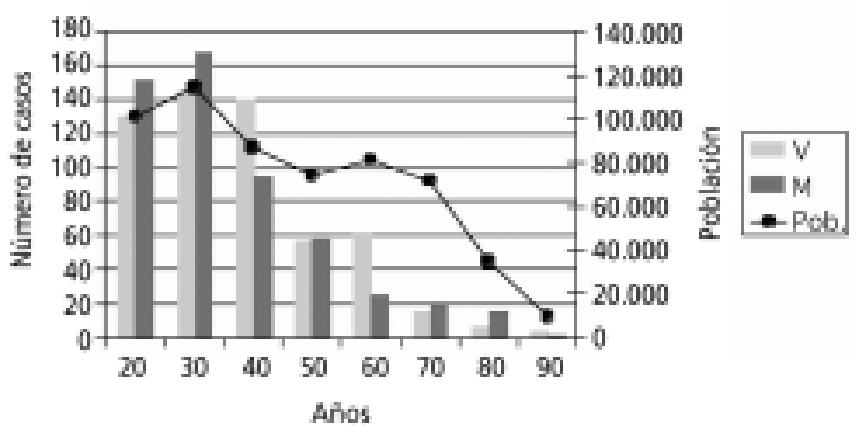

Fig. 1. Intoxicaciones: reparto numérico por edad y sexo. Se acompaña el Padrón del año 1996, del Área 11 del INSALUD.

\section{TIPO DE INTOXICACIÓN}

El número de casos de los diferentes tipos de intoxicación, su género, así como la media y la desviación estándar de las edades, figuran en la tabla I.

1. Intoxicaciones agudas voluntarias (IAV) $(150 / 100.000$ habitantes). El reparto por edades en cada tipo de IAV se muestra en la figura 2. Las medias de edad de uno y otro sexo en el conjunto de IAV son significativamente diferentes (error estándar: 0,90, p<0,001) y lo mismo cabe decir en las intoxicaciones alcohólicas (error estándar: 1,53, $\mathrm{p}<0,001)$, y las IAV por drogas $\mathrm{p}<0,05$, pero no en los restantes subgrupos.

2. Intoxicaciones accidentales (IAA) (19/100.000 habitantes). Los datos sobre edad y género se exponen en la tabla I. 
TABLA I

REPARTO SEGÚN TIPO DE INTOXICACIÓN Y SEXO

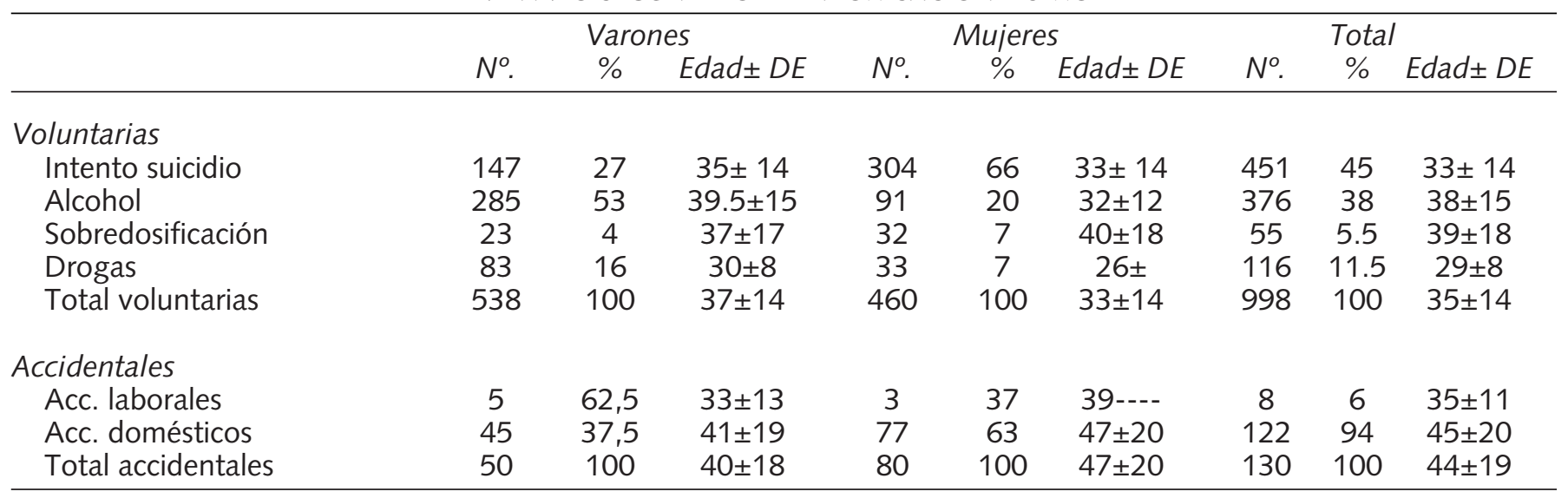

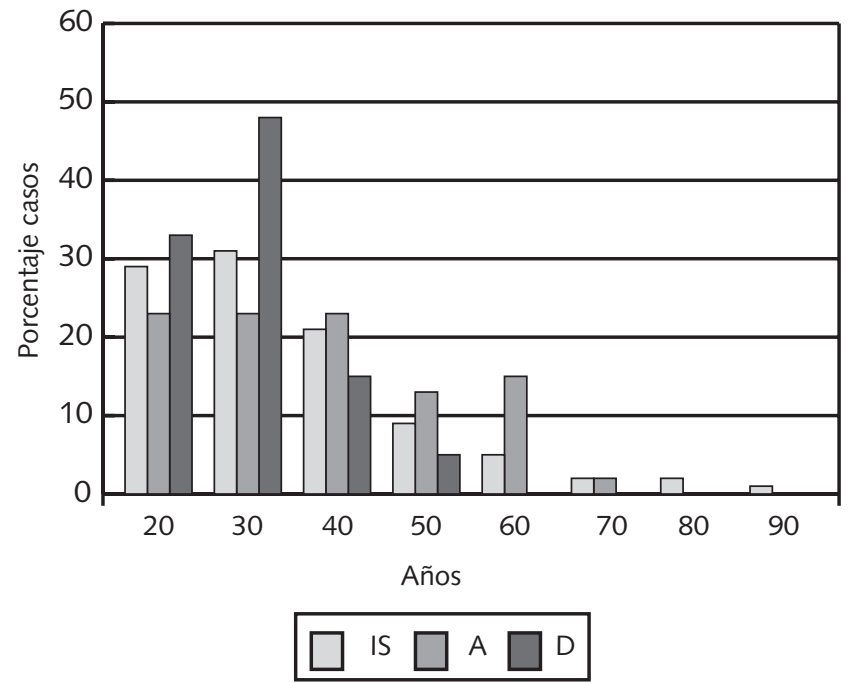

Fig. 2. Distribución por grupos de edad de los intentos de suicidio e intoxicaciones por drogas y alcohol.

\section{TÓXICOS IMPLICADOS}

1. Medicamentos: tóxico único en 375 casos (33\%) en el grupo general, que asciende hasta el $71 \%$ en los intentos de suicidio (IS) (79\% en las mujeres y $56 \%$ en los hombres). Los fármacos, su reparto conforme a los diferentes grupos terapéuticos y la incidencia según el género pueden verse en la tabla II. 56\% de las IA medicamentosas usaron solo un fármaco, $23 \%$ dos, $14 \%$ tres, $6 \%$ cuatro y $4 \%$ más de cuatro. Promedio en hombres 1,8 y 1,6 las mujeres.

La media de medicamentos por intoxicación es de 1,38 y el promedio de fármacos por presentación farmacéutica: 1,05.

2. No medicamentos: 632 casos 56\% (Tabla III).

3. Asociaciones de ambos 119 casos (11\%). En el $24 \%$ de las mezclas el no-fármaco era alcohol.

4. Desconocido: 2 casos, $0,1 \%$.
TABLA II

TÓXICOS IMPLICADOS: MEDICAMENTOS

Tóxicos: medicamentos Número \% Grupo \% Total

$\begin{array}{llll}\text { Benzodiacepinas } & 302 & 71 & 44\end{array}$

Antidepresivos

$84 \quad 19 \quad 12$

Neurolépticos

22

$5-3$

Barbitúricos

Otros psicofármacos

3

Total psicofármacos

16

5

1

4

$427 \quad 100$

1

2

00

62

Paracetamol

Salicilatos

Otros analgésicos

Aines

Total analgésicos

$\begin{array}{ccc}35 & 27 & 5 \\ 5 & 4 & 1 \\ 43 & 33 & 6 \\ 46 & 36 & 7 \\ 129 & 100 & 19\end{array}$

Otros fármacos

\begin{tabular}{lccc} 
Otros fármacos & 129 & --- & 19 \\
Desconocido & 2 & --- & 0 \\
\hline Totales & 687 & --- & 100
\end{tabular}

TABLA III

TÓXICOS IMPLICADOS: NO MEDICAMENTOS

\begin{tabular}{lc}
\hline Tóxicos: No medicamentos & $N^{\circ}$ casos \\
\hline Alcohol & $543(64 \%)$ \\
Cocaína & $67(8 \%)$ \\
Heroína & $41(5 \%)$ \\
Otras drogas & $52(6 \%)$ \\
Gases & $61(7 \%)$ \\
Cáusticos & $48(6 \%)$ \\
Otros tóxicos & $35(4 \%)$ \\
\hline Totales & $847(100 \%)$
\end{tabular}




\section{DISTRIBUCIÓN EN EL TIEMPO}

Ni el conjunto de la serie, ni los diferentes subtipos muestran diferencias significativas entre los días de la semana ni los meses del año.

Antecedentes: Un $85 \%$ de los pacientes con IS fueron valorados por un psiquiatra en la Urgencia, obteniéndose 148 antecedentes, a los que hay que sumar 212 procedentes de la anamnesis clínica, todos se enumeran en la tabla IV.

Destino: en la tabla V se detalla el destino de los pacientes según los diferentes tipos de intoxicación.

Discusión: la incidencia actual (170/100.000 habitantes), es un 5\% inferior a 1997 (6), esta disminución puede responder a un descenso ocasional o definitivo, pero en cualquier caso es un buen dato. La incidencia puede considerarse como intermedia con respecto a otras publicadas en nuestro país: Fuentes y cols. (13) (1985-86) 79/100.000, en Zaragoza; Cabo y cols. (16) (1988) 202/100.000, Elche (incluye pediátricas); Pérez y Belzunegui (14) (1989) 70/100.000, Navarra; Arruga y cols. (15) (1989-90) 195/100.000, Mataró; Querejeta (10) (1989-92) 45/100.000, Guipúzcoa; Cabo y cols. (16) (1990) 202/100.000 Alicante; Duce y cols. (20) (1995) 53/100.000 en Móstoles.

Edad y género: En 1994 (5) la media de edad de las mujeres disminuyó con respecto a series anteriores (2-4), incluso con significación estadística, y pasó a ser menor que la de los hombres y esta diferencia, aunque pequeña, se ha mantenido hasta la actualidad. La causa parece ser que los IS, que son la IAV más frecuente en las mujeres, son también más prevalentes en los grupos de edad más jóvenes (entre 15 y 34 -p<0,001 al comparar la media de edad con las de los restantes tipos de IAV-), mientras que en los hombres la IAV más frecuente es la etílica, predominante en edades mayores (25-44 años).
La proporción de los sexos en las cifras globales de la IA no ha cambiado en los últimos tres estudios (4-6) y se mantiene con un reparto al cincuenta por ciento. Al revisar los datos discordantes en series anteriores hemos observado que se debieron a predominios transitorios de determinadas intoxicaciones, y así, en la primera serie (2) predominaban los IS por lo que había más mujeres (63\%). En 1990 (4) predominaron las IA por alcohol y drogas y por ello, preponderaban los varones $(63,5 \%)$, mientras que en las tres ultimas series (4-6) se mantiene aproximadamente la proporción del tipo de intoxicaciones. También hemos observado cambios, aunque lentos y de no mucha amplitud en los IS de los varones que han pasado de ser uno de cada cinco en 1979 (2) a uno de cada tres en el 2000, asimismo en las intoxicaciones por drogas había una mujer por cada ocho casos en la observación inicial (2) y en la actualidad una por cada 3,5 y algo similar sucede con el alcohol.

\section{INTOXICACIONES VOLUNTARIAS}

1. Intento de suicidio: Nuestra serie actual parece indicar un descenso del número de IS, tras el progresivo ascenso desde los años $80(24,31,32)$ y partiendo de tasas muy inferiores a las de otros países -250/100.000 hombres y 240/100.000 mujeres en el Reino Unido en los años 80 y 314/100.000 hombres en Finlandia o 462/100.000 mujeres en Francia (10). Edad y género en los IS: La edad media para los IS es 33 años, similar a años anteriores (4-6) y a otras series (47), pero mayor que en 1979 (2) en que era de 27 años. Spicer y Miller (32) y Dieserud y cols. (33) describieron un predominio de mujeres, adolescentes y jóvenes entre 24 y 44 años. En nuestra serie se aprecia un envejecimiento de los hombres que

TABLA IV

El porcentaje expresa la proporción con respecto al encabezamiento

TABLA V

\begin{tabular}{|c|c|c|c|c|c|c|c|c|}
\hline \multicolumn{9}{|c|}{ TABLA V } \\
\hline \multicolumn{9}{|c|}{ DESTINO: REPARTO NUMÉRICO SEGÚN GÉNERO Y TIPO DE INTOXICACIÓN } \\
\hline & Varones & Mujeres & Voluntarias & I. suicidio & Alcohol & Drogas & Sobred. & Accidentes Total \\
\hline Alta domicilio & $352-60 \%$ & $205-38 \%$ & $434-45 \%$ & $40-9 \%$ & $296-79 \%$ & $75-65 \%$ & $23-42 \%$ & $123-95 \% 557-50 \%$ \\
\hline Valoración psiquiátrica & $200-34 \%$ & $311-58 \%$ & $510-51 \%$ & $385-85 \%$ & $63-17 \%$ & $31-27 \%$ & $31-56 \%$ & $1-1 \% \quad 511-45 \%$ \\
\hline Ingreso & $9-1 \%$ & $2-0.05 \%$ & $10-1 \%$ & $3-1 \%$ & $3-1 \%$ & $3-2 \%$ & $1-2 \%$ & $11-1 \%$ \\
\hline $\mathrm{UCl}$ & $16-3 \%$ & $8-1 \%$ & $22-2 \%$ & $13-3 \%$ & $4-1 \%$ & $5-4 \%$ & 0 & $24-2 \%$ \\
\hline Exitus & 2 & 1 & 3 & 1 & 1 & 1 & 0 & 0 \\
\hline Traslado & $11-2 \%$ & $14-2.5 \%$ & $22-2 \%$ & $10-2 \%$ & $10-2 \%$ & $2-2 \%$ & 0 & $25-2 \%$ \\
\hline
\end{tabular}

El porcentaje expresa la proporción con respecto al encabezamiento 

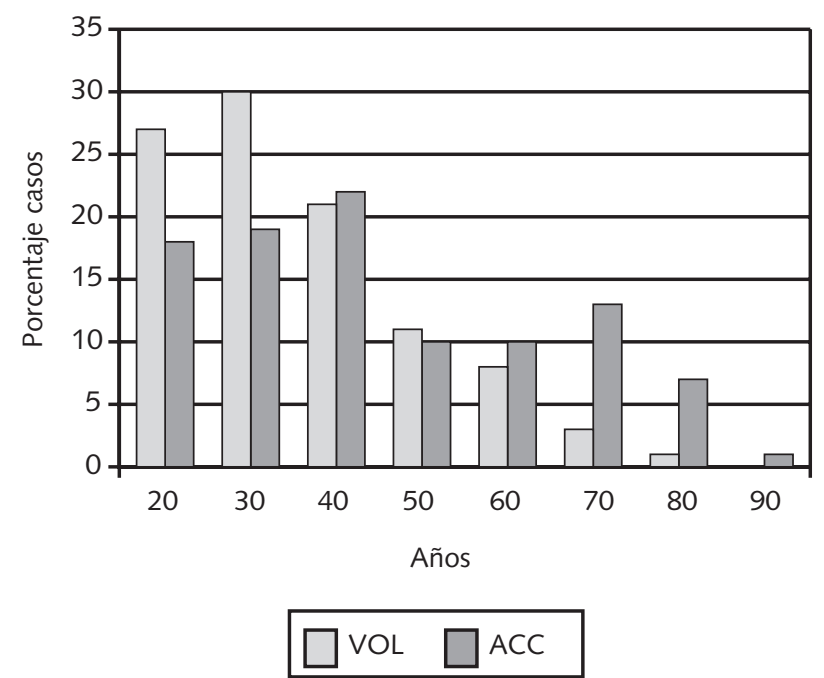

Fig. 3. Distribución por grupos de edad de las intoxicaciones voluntarias y accidentales.

hace que el grupo de 35-44 años sea desde 1997 (6) el más numeroso.

En la década de los 80 y primeros años de los 90 se detectó en el Reino Unido un aumento de IS en hombres (28), sin que quedase claro si concurría un descenso simultáneo en mujeres (34); en los veinte años que abarca nuestro estudio (26 ) han aumentado los varones y disminuido las mujeres casi en el mismo número (51 y 55 respectivamente). El IS en la mujer se asocia significativamente al deseo de llamar la atención sobre problemas de pareja o familiares, e implica una dificultad en el manejo de las habilidades sociales (18) y dado que aumentan los IS en los hombres (21) ¿puede asumirse esa misma problemática en los hombres?

Tóxicos en el intento de suicidio:

Medicamentos: Es habitual que en los IS la IA sea medicamentosa (93\% de nuestros casos, $94 \%$ en Dieserud y cols. (33) en Noruega 1984-95). Los más populares siguen siendo los psicofármacos si bien disminuyen un $12 \%$ con respecto al estudio del año anterior (6), básicamente por el descenso de benzodiazepinas, a pesar de lo cual el número de casos de la presente serie no difiere de otros datos publicados $(20,26,28)$. El resto de los psicofármacos no varía con respecto a las últimas series $(5,6)$. Los antidepresivos suponen una vez más el $12 \%$ del total, en línea con la mayoría de los publicados $(12,20,29)$ aunque Buckley y cols. (27) describen un mayor uso al comparar los datos de 1992 con los de 1987 y también Bialas y cols. (9) entre 1993 y 1998.

McLoone y Crombi $(36,37)$ describieron entre los años 1981 y 1991 descensos paralelos de las cifras de prescripción y el número de intoxicaciones con barbitúricos y benzodiazepinas y aumentos equivalentes en el caso de antidepresivos y antipsicóticos. Carlsten y cols. $(38,39)$ en sus series encuentran variaciones recíprocas entre las ventas de antidepresivos y el número de IS con ellos; cambio además selectivo ya que disminuyen ambos al considerar los tricíclicos y aumentan ambos en los ISRS. Sorprendentemente no encuentran paralelismo entre la disminución de la prescripción de benzodiazepinas y el número de IAV que causan.
El conjunto de IS por analgésicos sigue creciendo desde 1985 (3). La Aspirina en 1979 (2) causó 68 casos y en la actualidad sólo 5; simultáneamente el paracetamol no originó ninguna IA en 1979 (2) y ahora han sido 35 IA. En España el paracetamol, con un 5\%, carece de la importancia que tiene, como tóxico, en el Reino Unido -Bialas y cols. (9) $31 \%$ de todos los tóxicos en 1987-8 y crece hasta el $43 \%$ en 1992-93¿ocurrirá lo mismo en nuestro país?. Con respecto a los analgésicos la mayor evolución es la diversificación, hasta el punto que en nuestra publicación de 1997 (6) nos planteamos la conveniencia de abrir un epígrafe especifico de AINE que hoy se justifica al constituir casi un 40\% de los analgésicos.

Un $24 \%$ de los IS utilizaron asociaciones de fármacos y no-fármacos, cifra intermedia respecto a otras publicadas [Duce y cols. (20) 20\%, Verstraete y Buylaert (31) 36\%]. Un $80 \%$ de estos casos incluían alcohol como no-fármaco y el $20 \%$ restante drogas y venenos tradicionales.

Tipología del IS: (antecedentes):

Se han recogido 360 antecedentes, cifra mayor que otras publicadas (21). Se diagnosticó depresión al 33\%, (las tres cuartas partes mujeres) y el $18 \%$ admitieron intentos previos, cifras sensiblemente iguales a las de años anteriores $(5,6)$ y a otras publicadas (9). Las toxicomanías, tanto alcohol como drogas, son un importante factor de riesgo para el IS $(33,40)$ y tiene mayor relevancia en el subgrupo de varones, ya que hasta un $20 \%$ de ellos eran adictos a drogas o alcohol; esta observación ya se realizó en estudios anteriores pero la proporción ha aumentado [1990 (4): 6\%, 1994 (5): 4\% y 1997 (6): 4\%).

Destino de los IS:

Un $1 \%$ ingresó en planta y $3 \%$ en UCI frente al $2 \%$ de la serie general, cifra intermedia entre otras. Hubo tres fallecimientos $(0,3 \%)$.

Características de los Ingresos en UVI por IS:

Los tóxicos no presentan diferencia estadística con los del grupo general -salvo en el caso de la heroína-, tampoco hubo más asociaciones de tóxicos. El 58\% de este subgrupo eran toxicómanos (alcohol y/o drogas) y habían realizado intentos previos el $12 \%$.

2. Intoxicaciones etílicas: En el año 2000 disminuyen un $11 \%$ con respecto a 1997 , una parte, al menos, explicada por la asistencia prehospitalaria (22) pero no debemos olvidar que si comparamos estas cifras con las de nuestra primera serie (2) se han triplicado.

Edad y género: La disminución del número descrito se produce en los hombres ya que el número de mujeres es igual en ambos años. Los integrantes del grupo de IA etílicas también parecen hacerse mayores si nos atenemos a las medias de edad, y además, sorprendentemente, disminuyen los ingresos en el grupo más joven (15-25 años) y concretamente en mujeres disminuye un $39 \%$, ¿debido a una mejor asistencia extrahospitalaria? En otros estudios se han encontrado cifras más altas de jóvenes $40 \%$ (22). El cambio en la composición de los grupos de edad de 45-54 y 55-64 está artefactada por los repetidos ingresos de un varón que ha cambiado de grupo etario con respecto a la serie del año anterior. De modo similar a lo publicado por Bugarin y cols. (22), cuando separamos los casos con y sin alcoholismo encontramos que los segundos son más jóvenes (sobre todo el grupo de mujeres $\mathrm{p}<0,05$ ), que acuden el doble los fines de semana (principalmente los hombres) mientras que en los alcohólicos no existe esta diferencia ni en cuanto al día ni en cuanto al género, lo cual haría paralelos la edad y el etilismo crónico. Se han recogido 3 casos de 
alcohólicos en menores de 25 años, todos ellos varones, hay general acuerdo que el alcoholismo empieza a aparecer a partir de los 25 (41). En el grupo de alcohólicos encontramos algunos casos de depresión, drogadicción e intentos previos de suicidio. Entre los no alcohólicos hay el doble de casos de depresión que en los alcohólicos, mientras que drogadicción o intentos previos son idénticos. Hubo un fallecimiento en este grupo que supone $0,26 \%$ de las etílicas.

3. Intoxicaciones por drogas: Las intoxicaciones por drogas experimentan un aumento porcentual notable con respecto a años anteriores (8 veces más que en 1979) (2) de tal manera que mientras que en la primera serie (2) representaban el $2 \%$ de las IAV, ahora son el $12 \%$, a pesar de este trascendental aumento, estamos lejos de los 285 casos de 1990 (4), época del boom de la heroína $(7,15)$.

La edad de las mujeres es significativamente menor que la de los hombres, pero en ambos la edad media progresa año tras año (2-6). Este tipo de IAV se mantiene como dominio habitual de varones, pero en la serie actual el mayor crecimiento porcentual -que no numérico-corresponde a las mujeres que pasan del $13 \%$ (2) al 28\% y aumentan en estos veinte años de 2 a 33 casos. También hemos observado que en los últimos años existe un incremento del grupo más joven ¿implica eso una sustitución de una forma de drogarse por otras?, ¿cambia la heroína intravenosa por otras drogas y otras vías?, parece claro que el miedo al sida contribuyó a acabar con el consumo parenteral de heroína, pero las drogas siguen consumiéndose aunque de una forma diferente: la heroína ha quedado estancada en los valores de 1994 (4), seguramente porque ahora se consume en asociación con cocaína y también por ello ésta ha pasado de 13 casos (5) a 67. Si tenemos en cuenta que en 1994 (5) no hubo ninguna asistencia por intoxicación con MDMA, que en 1997 (6) se atendieron 5 casos y en el 2000 (14), podemos deducir que en los próximos años sufrirá un crecimiento geométrico. En 1994 Rodríguez y cols. (19) encontraron que las IA por alucinógenos, anfetaminas y otros estimulantes, eran cien veces menos que las de opioides o cocaína, apenas ocho años después encontramos que ahora representan una quinta parte, a pesar de lo cual nuestra incidencia 3/100.000 habitantes es menor que la suya (entre 3.4 y 9,4/100.000 habitantes). Estos autores (19) explican que cabe la posibilidad de que queden ocultas por la mentira del paciente y el desconocimiento del médico, sin que éste sea consciente de su existencia. ¿Puede ser ésta la explicación de que sólo la mitad de los intoxicados por drogas sean drogadictos? Si a esta cifra le sumamos el $8 \%$ de alcohólicos resulta que de las intoxicaciones por drogas solo un $61 \%$ son toxicómanos. La valoración psiquiátrica permitió encontrar cuatro casos de depresión y un intento previo de suicidio. El destino de los pacientes intoxicados por drogas fue: alta $65 \%$, alta previa valoración psiquiátrica $27 \%$ y cuatro ingresaron en UVI por lo que las drogas causaron el $17 \%$ de los ingresos en UCI y dos en planta, un intoxicado por una mezcla de heroína y benzodiazepinas fue exitus $(0,86 \%)$.

4. Sobredosificaciones: En el año 2000 hay 55 casos incluidos en este ítem lo que supone un aumento apreciable ya que hubo 37, en 1997 (6). Este aumento no puede atribuirse a la taxonomia ya que no ha cambiado la persona que las juzga como tales, pero las características de la sobredosis no son idénticas a ninguno de los restantes tipos de IAV ni accidentales y es frecuente que haya variaciones poco explicables. La mayoría son mujeres, como es habitual, y la edad media en torno a los 40 años (37 los hombres y 40 las mujeres) aunque hay casos desde los 15 a los 90 años. Sólo un 6\% de estos pacientes correspondían a otras áreas sanitarias, la cifra más baja de toda la serie.

Como es habitual los tóxicos de este epígrafe son medicamentos, la mayoría de los cuales poseen gran eficacia en el tratamiento sintomático (ansiolítico $-46 \%$ - y analgésico $-11 \%$-) o bien el paciente los mezcla con otros medicamentos para potenciar su efecto (1,3 de promedio, 1,6 en los IS) incluso con alcohol (7\%). En esta ocasión se incluyen diez pacientes con depresión intoxicados por antidepresivos y dos drogadictos que consumieron un exceso de metadona en un intento de aplacar el síndrome de abstinencia.

No falleció ninguno de estos pacientes, ni precisó ingreso en UVI, uno ingresó en planta por la intoxicación y a 31 (56\%) se les pidió valoración psiquiátrica intra-urgencia.

Intoxicaciones accidentales: Representan solo el $11 \%$ del total de la serie. La edad media no se ve influida por el género de modo significativo. La mayoría son accidentes domésticos $94 \%$.

Accidentes laborales: Se atendieron 8 pacientes con intoxicaciones laborales, la mayoría por gases de combustión -uno de ellos precisó ingreso en UCI- y el resto cáusticos y detergentes. Son más jóvenes que los pacientes por accidentes domésticos.

Accidentes domésticos: Es común que en este apartado haya más mujeres $63 \%$ y que sean mayores -media 47añosque los hombres -media 41 años-. El 70\% de los hombres eran menores de 45 años, mientras que las mujeres de ese grupo de edad eran solo el 50\%. Existe una distribución de los casos con respecto a la edad bimodal como ya hemos descrito con anterioridad (3-5). El grupo de tóxicos más frecuentes ha sido los gases de combustión en incendios $45 \%$, seguidos de los cáusticos $32 \%$. Un caso de accidente doméstico precisó ingreso en UCI.

\section{Bibliografía}

1. Caballero PJ, Dorado MS. Factores epidemiológicos de la intoxicación. Medicine (Madrid) 1980; 59: 3691-3699.

2. Caballero PJ, Dorado MS, Alonso F. Intoxicación aguda: Estudio de 673 casos. Med Clin (Barc) 1981; 77: 139-145.

3. Caballero PJ, Gutiérrez F, Dorado MS. Epidemiología de la intoxicación aguda: Estudio comparativo 1979-1985 en el área sur de la Comunidad Autónoma de Madrid. Rev Clin Esp 1987; 181: 334-339.

4. Dorado MS, Álvarez R, Caballero PJ, Medina J, Casanova C, Granado JA. Epidemiología de la intoxicación aguda: Estudio de 815 casos habi-

dos en 1990 en el área sur de la Comunidad de Madrid. Rev Clin Esp 1992; 191: 131-136.

5. Dorado MS, Martín J, Sabugal G, Caballero PJ. Epidemiología de la intoxicación aguda: estudio de 613 casos habidos en 1994 en el área sur de la Comunidad de Madrid. Rev Clin Esp 1996; 196: 150-156.

6. Caballero PJ, Dorado MS, Brusint B, Jerez B, Medina M: Vigilancia epidemiológica de la intoxicación aguda 1997 (Estudio de 1.140 casos del área sur de la Comunidad de Madrid). Rev Clin Esp 1999; 199: 424430. 
7. Muñoz J. y Guerrero JE. Intoxicaciones: panorama actual. Rev Clin Esp 1996; 196: 141-142.

8. Wolfle J. Kowalewski S. Epidemiology of ingestion in a regional poison control center over twenty years. Vet Hum Toxicol 1995; 37: 367-8

9. Bialas MC, Reid PG, Beck P, et al. Changing patterns of self-poisoning in a UK health district. QJM 1996; 89: 893-901

10. Schmidtke A. Bille-Brahe U. DeLeo et al. Attempted suicide in Europe: rates, trends and sociodemographic characteristic of suicide attempters during the period 1989-1992. Results of the WHO/EURO Multicentre Study on parasuicide. Acta Psychiatr Scand 1996; 93: 327-338.

11. Dorado S. Variaciones epidemiológicas en las intoxicaciones agudas voluntarias atendidas en el hospital Doce de Octubre de Madrid desde 1979 a 1994. 1998. Tésis doctoral. Universidad Complutense de Madrid.

12. Bundó M, Fandós JM, Diez J, Ferran M, Mundet X, Ibáñez L: Intoxicación voluntaria farmacológica en Barcelona: 110 casos. Atención Primaria 1987; 4: 402-6,

13. Fuentes J, García-Galán A, Muñoz JR et al. Intoxicaciones agudas en la unidad de urgencias de un hospital general. Arch Fac Med Zaragoza 1990; 1: 27-30.

14. Pérez JM, Belzunegui T. Intoxicación aguda voluntaria en Área de salud Navarra I: Rev. Sanid. Hig. Publica (Madrid) 64 7-8 401-14 1990.

15. Arruga C. Almirall J, Bassa J Balanzó X. Intoxicaciones agudas en un hospital comarcal. Med Clin (Barc) 1992; 98: 117-118.

16. Cabo M, Marti JB, Miralles S, Marti JL. Etiology of intoxication: a study of 557 cases. Eur J Epidemiol 1993; 4: 361-7.

17. Bajo A, Santos ME, Sanz F, et al. Estudio epidemiológico sobre intoxicaciones agudas y dotaciones de botiquines de antídotos. An Med Interna (Madrid) 1999; 16: 285-9.

18. Marín Lozano et al. Tentativas de suicidio en la urgencia del hospital general: un estudio epidemiológico y clínico. An Psiquiatría (Madrid) 1995; 1: 259-263.

19. Rodríguez M A, Barrio G, De la Fuente L et al: Urgencias relacionadas con el consumo de drogas, alucinógenos y anfetaminas atendidas en quince hospitales españoles durante 1994. Rev Clin Esp 1997; 197: 804-809.

20. Duce S., López E., Navas V. et al: Intoxicaciones medicamentosas voluntarias atendidas en un Servicio de Urgencias. Emergencias 1998; 10: $225-233$.

21. Tejedor MC, Díaz AM, Álvarez E, Castillón JJ, Pericay JM. Intento de suicidio: Cambios epidemiológicos entre 1969 y 1996. Estudio retrospectivo de 1.150 casos. Actas Esp Psiquiatr 1999; 27: 292-7.

22. Bugarin R, Galego P, Gude F, García A, Galban C: Estudio de las intoxicaciones etílicas agudas en un servicio de urgencias. An Med Interna (Madrid) 2000; 17: 588-91

23. Carpintero JM, Ochoa FJ, Ruiz JI, Bragado L, Palacios G, RamalleGómara E, Grupo de Toxicología de Semes-La Rioja. Prevalencia de las intoxicaciones agudas en Urgencias de La Rioja. Emergencias 2000; 12 : 92-97.

24. PROSEREME (Programa Selectivo de Revisión Fármaco-terapéutica de los Medicamentos Españoles) Dirección General de Farmacia y Productos Sanitarios. Ministerio de Sanidad y Consumo. Madrid, 1981.

25. Kelly CB, Weir J, Rafferty T, Galloway R. Deliberate self-poisoning presenting at a rural hospital in Northern Ireland 1976-1996: relationship to prescribing. Eur Psychiatry 2000; 15: 348-53

26. Kapur N, House A, Creed F, Feldman E, Friedman T, Guthrie E. General hospital services for deliberate self-poisoning: an expensive road to nowhere? Postgrad Med J 1999; 888: 599-602.

27. Buckley NA, Whyte IM, Dawson AH, McManus PR, Ferguson NW. Self-poisoning in Newcastle, 1987-1992. Med J Aust 1995; 162: 174175.

28. Beck P, Lazarus J, Scorer R, Smith P, Routledge P. Increasing rate of deliberate self poisoning. BMJ 1994: 308: 789.

29. Blanc PD, Jones MR, Olson KR. Surveillance of poisoning and drug overdose through hospital discharge coding, poison control center reporting, and the Drug Abuse Warning Network. Am J Emerg Med 1993; 11: 14-19.

30. World Health Organisation. Health-for-all targets. The health policy for Europe. Summary of the update edition, September 1991. Copenhagen: WHO EUR ICP/HSC 013,1992.

31. Verstraete AG y Buylaert WA: Survey of patients whith acute poisoning seen in the Emergency Department of the University Hospital of Gent between 1983 and 1990 Eur J Emerg Med 1995; 4 : 217-23.

32. Spicer RS, Miller TR: Suicide acts in 8 states: incidence and case fatality rates by demographics and method: Am J Public Health 2000; 90: 1885-91.

33. Dieserud G, Loeb M, Ekeberg O. Suicidal behaviour in the municipality of Baerum, Norway: a 12-year prospective study of parasuicide and suicide: Suicide Life Threat Behav 2000; 30: 61-73.

34. Stark C, Smith H, Hall D. Increase in parasuicide in Scotland (letter comment) BMJ 1994; 308: 1569-1570.

35. Carter GL, Whyte IM, Ball K, et al, Repetition of deliberate self-poisoning in an Australian hospital-treated population. Med J Aust 1999; 170: 307-11.

36. Mcloone $\mathrm{P}$, Crombie IK. Hospitalisation for deliberate self-poisoning in Scotland from 1981 to 1993 : Trends in rates and types of drug used. $\mathrm{Br}$ J Psychiatr 1996; 169: 81-85.

37. Crombie IK, McLoone P. Does the availability of prescribed drugs affect rates of self poisoning? Br J Gen Pract 1998; 433: 1505-6.

38. Carlsten A, Allebeck P, Brant L. Are suicide rates in Sweden associated with changes in the prescribing of medicines? Acta Psychiatr Scand 1996; 94: 94-100.

39. Carlsten A, Waern M, Allebeck P. Suicides by drug poisoning among the elderly in Sweden 1969-1996. Soc. Psychiatry Psychiatr Epidemiol 1999; 34: 609-14

40. Soukas J y Lönnqvist J. Suicide attempts in which alcohol is involved: a special group in general hospital emergency rooms. Acta Psychiatr Scand 1995: 91: 36-40.

41. Del Río Mc, Consumo de alcohol y problemas relacionados con el alcohol en España. Jano 1998; 54: 197-203. 